\title{
Are donkey sentences and bare conditionals family or friends?
}

Evidence from $\mathrm{L}_{1}$ Chinese

\section{[驢子句和光桿句是家人還是朋友?華語為第一 語言的佐證]}

\author{
Wei-ling Eileen Lin [林韋伶], \\ Chun-yin Doris Chen [陳純音] and \\ Gerardo Fernandez-Salgueiro [颯楊] \\ National Taiwan Normal University [國立臺灣師範大學]
}

The present study investigates children's first language acquisition of donkey sentences and bare conditionals in Mandarin Chinese, both of which are concerned with quantification. Kindergarteners, Grade 2 and Grade 4 were recruited for experimental groups, each group consisting of 18 subjects, and 18 adults comprised a control group against which to compare their interpretations. Each subject finished two Truth-Value Judgment tasks, which were sentences in isolation and sentences in context. The results of this research identified a developmental pattern regarding the acquisition of donkey sentences and bare conditionals in Mandarin Chinese. It was found that overall children under seven years of age had difficulty interpreting quantificational sentences. First, concerning the relatedness of the two constructions, all four groups showed a tendency to find donkey sentences easier to interpret than bare conditionals. With respect to contextual effects, by Grade 2, children could obtain adult-like interpretations of donkey sentences in a biasing context, but it was not until they were in Grade 4 that they could interpret both donkey sentences and bare conditionals in their supporting context with adult-like readings. As a result, the subjects' interpretations were greatly affected by context, but the two constructions were affected in different ways.

Keywords: donkey sentences, bare conditionals, first language acquisition, quantification, Mandarin Chinese

關鍵詞：驢子句、光桿句、第一語言習得、量化、華語 


\section{Introduction}

When it comes to first language acquisition, the Innateness Hypothesis (Chomsky 1986, Cook 1988, Gopnik 1988, Keil 1989, Gelman \& Wellman 1991, Gopnik \& Meltzoff 1997) is one of the most crucial theories to date. It argues that children are born with some principles of language that enable them to master a language within a short period of time. Moreover, not all linguistic knowledge is acquired simultaneously. It has been found that children can comprehend certain concepts more easily and quickly than others, and that they acquire all linguistic properties on the basis of easier ones. In other words, there is a sequence in mastering language, and the acquisition of quantification is one case that can be investigated.

It has been found that in the process of acquisition children have difficulty comprehending quantificational sentences (Herburger 1997, Cohen 2001, Geurts 2003). For example, for a sentence like "Every kid holds an umbrella," they tend to be interpreted as "Every umbrella-holder is a kid." A donkey sentence, which gets its name from the original example, is one construction worth studying in terms of acquisition. Sentence (1), where the pronoun it refers back to the antecedent a donkey, can be interpreted as "Every farmer who owns a donkey beats at least one of the donkeys that he has," indicating that there is at least one donkey in the world involved, that is, an existential reading of the sentence.

(1) Every farmer who owns a donkey beats it.

(Geurts 2002: 129)

On the other hand, (1) can also be correctly interpreted as "Every farmer who owns a donkey beats all of the donkeys that he has," implying that all of the donkeys are involved, yielding a universal reading. Both readings for (1) are possible, but it has been found that speakers tend to have a preference for one of them. For example, if a sentence is headed by a universal quantifier, like every, a universal reading is more likely to be favored, but if it is headed by an existential quantifier, like some, an existential reading tends to be chosen (Kanazawa 1994, Geurts 2002, Foppolo 2009).

Like donkey sentences, Chinese conditional sentences as shown in (2)-(4) are also concerned with an interplay of logic between quantifiers and variable pronouns:

(2) Shei xian lai, shei xian chi.

who come first who first eat

'If X comes first, $\mathrm{X}$ eats first.'

(Cheng \& Huang 1996: 127) 
(3) Ruguo you shei qiao men, ni jiu jiao ta jin-lai. if have who knock door you then ask him/her come-in 'If someone knocks on the door, you'll ask him/her to come in.'

(Cheng \& Huang 1996: 142)

(4) Ni jiao shei jin-lai, wo dou jian ta. you ask who come-in I all see him/her 'Whoever you ask to come in, I will see him/her.' (Cheng \& Huang 1996: 142)

Sentence (2), an example of a bare conditional, is different from ordinary conditional sentences such as (3) and (4) in that neither a conjunction, like ruguo 'if' in the antecedent clause as in (3), nor an adverb, like dou 'all' in the consequent clause as in (4), is present. As can be seen in (2), the variable, the wh-word shei 'who', in the antecedent clause, shei xian lai 'who comes first,' is connected to shei 'who' in the consequent clause shei xian chi 'who eats first', meaning that whoever comes first eats first.

There have been debates over whether Chinese bare conditionals can be regarded as a type of donkey sentence (Cheng \& Huang 1996, Pan \& Jiang 1997, Cheung 2007, Wang 2007) since the two constructions have referentiality in readings in common. Some argue that the two constructions are related (Cheng \& Huang 1996, Pan \& Jiang 1997) while others deny this (Wang 2007). In an English donkey sentence like (1), two approaches are employed to explain the donkey pronoun $i$, which is anaphoric to a donkey.

The first approach considers it to be an E-type pronoun where "E" stands for "existential," meaning the pronoun it expresses an existential property of the antecedent $a$ donkey, yielding a meaning that at least one of the donkeys (but not necessarily all the donkeys) owned by a farmer is beaten by him.

The other approach is the unselective binding approach, which indicates the existence of an implicit operator (called the necessity operator, NEC) that unselectively binds two anaphoric nouns (it and a donkey). This operator serves as a binder and licenser that not only binds the two variables in the sentence but also licenses a quantificational force for the variables, as can be seen in (5).

(5) NEC $[\mathrm{x}, \mathrm{y}][\operatorname{farmer}(\mathrm{x}) \text { and } \operatorname{donkey}(\mathrm{y}) \text { and own }(\mathrm{x}, \mathrm{y}) \rightarrow \operatorname{beat}(\mathrm{x}, \mathrm{y})]^{1}$

(Pan \& Jiang 1997)

Hence, following this approach, for (1), both the pronoun it and the antecedent a donkey are bound by the operator (NEC), which is the quantifier every that gives quantificational force to the variables. Therefore, operator binding results in ambiguity: an existential reading (i.e., for a farmer who has a donkey, there

1. This formula is taken from Pan \& Jiang (1997) but with slight changes to fit it to sentence (1). 
exists at least one donkey that is beaten by him) and a universal reading (i.e., for a farmer who has a donkey, all of the donkeys are beaten by him).

It has been argued by Cheng \& Huang (1996) that the above two approaches to donkey sentences account for conditionals in Mandarin Chinese. For them, bare conditionals are a group of conditionals interpreted through the unselective binding approach, and other conditionals are interpreted through the E-type pronoun approach. Bare conditionals can only take a $w h$-word in a consequent clause as in (6), while ruguo- and dou-conditionals can allow either an overt or covert pronoun in the consequent clause, but not a wh-word, as shown in (7) and (8): ${ }^{2}$

(6) Shei lai shei/ta/[e]/na-ge-ren jiu you liwu. who come who/he(she)/[e]/that-CL-person just have present 'Whoever comes will get a present.'

(7) Ruguo ni kandao shei, qing jiao *shei/ta/[e]/na-ge-ren lai jian if you see who please tell who/him(her)/[e]/that-CL-person come see wo.

me

'If you see someone, please ask that person to come see me.'

(Cheng \& Huang 1996: 131)

(8) $\mathrm{Ni}$ jiao shei jinlai wo dou jian *shei/ta/[e]/na-ge-ren.

you ask who enter I all see who/him(her)/[e]/that-CL-person

'Whoever you ask to come in, I'll see him/her.' (Cheng \& Huang 1996:130)

Cheng \& Huang (1996) propose that the two types of conditionals are in a complementary distribution where bare conditionals adopt the unselective binding to interpret their references while the other two conditionals take the E-type pronoun approach.

The approaches are selected according to whether or not there is a wh-word in the consequent clause of a conditional sentence. For bare conditionals, which in principle require that no overt elements like ruguo 'if' or dou 'all' appear in the clauses, a $w h$-word in the consequent clause can only be taken to be anaphoric to the $w h$-word in the antecedent clause. This is illustrated below:

(9) $\mathrm{Ni}$ xihuan shei, wo jiu piping shei $/{ }^{*}$ ta $/{ }^{\star}[e] /{ }^{*}$ na-ge-ren.

you like who I then criticize who/he(she)/[e]/that-CL-person

'If you like X, I then criticize X.'

(Cheng \& Huang 1996: 128)

2. Nevertheless, some native speakers of Chinese do not find it ungrammatical with the use of $w h$-words in the consequent clause in (7) and (8). 
Hence, in a bare conditional like (9), only shei 'who,' but not a pronoun ta 'he/ she, an empty category, or a definite noun phrase na-ge-ren 'that-CL person' can appear in the consequent clause. Based on this, Cheng and Huang argue that bare conditionals can be analyzed by the unselective binding approach since the two wh-words in the antecedent and the consequent clauses are both bound by the necessity operator. With the adoption of the unselective binding approach, it is proposed that the readings of bare conditionals are universal. Accordingly, they believe that since the approaches adopted to analyze donkey sentences are also available to analyze bare conditionals, the two constructions are related.

Following Cheng \& Huang (1996), Pan \& Jiang (1997) also deem bare conditionals to be a type of donkey sentence. ${ }^{3}$ For them, bare conditionals, like donkey sentences, exhibit anaphoric properties. In addition, the approaches used to analyze donkey sentences can be used to analyze bare conditionals as well; hence, the term "family" used in the title above.

However, Wang (2007) disagrees with the idea that donkey sentences and bare conditionals are related. For him, a bare conditional is actually a relative clause sentence with identical $w h$-words anaphoric to each other where the first clause takes the role of a relative clause and the second clause is a matrix clause, as in (10), extracted from Wang (2007:71):
a. Sheme pianyi, ta
mai sheme.
what cheap he(she) buy what
b. Pianyi de dongxi ta dou mai. cheap DE thing he(she) all buy 'He/She buys anything that is cheap.'

3. Although Pan \& Jiang (1997) also state that conditionals are related to donkey sentences, they argue that both bare conditionals and ruguo- and dou-conditionals can take either overt pronouns or $w h$-words in the consequent clause, and the two approaches can be adopted to analyze conditionals. Hence, they claim that there is no such complementary distribution of conditionals as Cheng \& Huang (1996) proposed. According to Pan \& Jiang (1997), the adoption of different approaches renders different readings of conditionals. The E-type pronoun approach imposes on the sentence a restriction that there is only one individual or entity to make the proposition tenable, while the unselective binding approach offers a universal reading. The following are readings for "Shei xian lai, shei/ta xian chi" adopted with the unselective binding approach, as stated in (i), and with the E-type pronoun approach, as in (ii), both of which are taken from Pan \& Jiang (1997:13).

i. NEC $[\mathrm{x}][\operatorname{come}(\mathrm{x})$ first] [eat (x) first] (universal reading)

ii. a. NEC $[\mathrm{x}, \mathrm{s}][\operatorname{come}(\mathrm{x})$ first in $\mathrm{s}]$ [eat(x) first in s] (existential reading)

b. NEC $[\mathrm{s}]\left[\mathrm{H}_{\mathrm{x}} \operatorname{come}(\mathrm{x})\right.$ first in $\left.\mathrm{s}\right]$ [eat(he/she) first in $\left.\mathrm{s}\right]$ (existential reading) 
In (10a), the first wh-word sheme 'what' is considered to be a relative pronoun while the second behaves like the head of the relative clause. Examples (10a) and (10b) denote the same meaning, "He will buy whatever is cheap."

In addition, Wang argues that in (10a) only the first $w h$-word helps to trigger a universal reading (i.e., sheme pianyi 'anything cheap') while the second is only a component anaphoric to the first one (i.e., the head sheme 'what' anaphoric to sheme pianyi). The differences between the two $w h$-words can also be found phonetically. It is claimed that only the first $w h$-word can be stressed in the sentence. However, the universal reading is obtained by the presence of dou 'all' in (1ob).

All in all, of these three previous studies, two have argued for the familyhood of donkey sentences and bare conditionals (Cheng \& Huang 1996, Pan \& Jiang 1997), and the other has disputed such an analysis (Wang 2007). Hence, the present study attempts to find out whether or not the two constructions are related by providing empirical evidence from Chinese children's interpretation of these two types of sentence.

In addition, according to Prévost \& Paradis (2004), Foppolo (2009), and Ireri et al. (2012), context can affect interpretations of sentences involving a syntaxsemantic interface. It has been found that children depend on context to infer the meanings of sentences, and that context influences interpretations of quantification as well. Accordingly, the effect of context on the two constructions will also be discussed.

Since such a small amount of the literature (Crain et al. 2009) is concerned with $\mathrm{L}_{1}$ acquisition of donkey sentences and bare conditionals, the present study aims to provide clues to the relatedness of the two constructions and to the factors that may influence their readings through comparing children's and adults' interpretations of these sentences.

In order to investigate whether theoretical approaches are the mechanism operating in children's acquisition, we will address the following research questions:

(11) a. Are bare conditionals related to donkey sentences in terms of their interpretations?

b. Is there a contextual effect on the donkey construction and the bare conditional construction?

c. At what age do children obtain an adult-like interpretation of donkey sentences and bare conditionals? 


\section{Literature review}

Since there is no experimental research on Chinese donkey sentences and bare conditionals, three studies on English donkey sentences are reviewed in chronological order (Geurts 2002, Foppolo 2009, Grosz et al. 2014) in this section.

\subsection{Geurts (2002)}

Geurts (2002) aimed to illustrate the interplay between quantifiers and world knowledge through an experiment, and objected to the strong claim of judging the interpretations of donkey pronouns as having a direct and arbitrary relation with quantifiers. The subjects in the experiment were twenty native Dutch speakers who were recruited to interpret 24 Dutch donkey sentences along with 75 fillers. Since there is no difference between English and Dutch donkey sentences, the Dutch test items were simply translations of English versions. Knowing that descriptions would offer biasing information to the interpretations, Geurts conducted a Truth-Value Judgment task by presenting each test item with two pictures and asking the subjects to judge whether the target donkey sentence could conform to them. Four quantifiers ${ }^{4}$ were utilized - every, not every, some and nowith six different situation type settings for the donkey sentence test items.

It turned out that for sentences with the quantifiers some and no, nearly all the subjects accepted an existential reading in all six situations, but for sentences with the universal quantifiers every and not every, the interpretations did not display a neat consensus. In general, the $60 \%$ preference for universal readings showed that with universal quantifiers, there was an overall tendency for universal readings; however, contextual influence was found to lower the preference for universal readings.

As a result, Geurts proposed that the interpretation of donkey sentences relies on the initial quantifier and world knowledge, and that donkey sentences do not have definite interpretations. Geurts' analysis is quite promising, offering us factors that influence interpretations, including world knowledge and the nature of both quantifiers and indefinite nouns.

\subsection{Foppolo (2009)}

Foppolo (2009) aimed to test whether there is a preferred interpretation of the dependent variable in donkey sentences, and whether there is a default one

4. Geurts named every, not every, some, and no as determiners. For the sake of consistency, we call them quantifiers in the present study. 
that retains the monotonicity of the quantifier based on the generalization of Kanazawa (1994), which states that a universal quantifier entails a universal reading of the sentence while an existential quantifier leads to an existential reading.

Two experiments were employed by Foppolo (2009) to explore whether a default reading existed, whether the preference was in the same vein of Kanazawa's analysis, and whether biasing contexts influenced the reading. In the first experiment, thirty subjects were asked to perform a Truth-Value Judgment task and to evaluate whether any of the four pictures could express the interpretation of the target sentence with a quantifier. Target sentences with three quantifiers, every, no and some, were put into three different situations - one that was compatible with both universal and existential readings (non-differentiating true, NDT), one that was compatible with neither of the two readings (nondifferentiating false, NDF), and one that was compatible with only one of the readings (differentiating-critical, DC). The results showed that a default interpretation existed. For sentences with no and some, the subjects tended to interpret them with an existential reading, but there was no clear tendency for sentences using every. Fopplo's first experiment showed that Kanazawa's Generalization was partially correct. The second experiment was employed to further confirm whether the alternative readings were accessible. The subjects were another 36 students recruited to examine the test items in a Truth-Value Judgment task. By investigating only the NDF and the DC conditions, and providing biasing contexts, Foppolo explored whether the subjects could be induced to choose an alternative reading, which would be a disfavored one, and the two situations were designed with the disfavored reading based on the generalization. No significant contextual effect was found in the control situation. However, a significant effect was presented in the DC condition for every in that, with biasing contexts, the subjects were greatly influenced and chose to take an existential reading, the disfavored one. On the other hand, biasing contexts did not cause the subjects to change their interpretation donkey pronouns in sentences with no or some.

\subsection{Grosz et al. (2014)}

Grosz et al. (2014) employed an experiment concerning the anaphoric properties of donkey sentences involving constraints on the antecedent. One hundred and twenty-five adult subjects were recruited. An acceptability-rating task was conducted on a total of 102 sentences of which 30 were critical sentences and 72 were fillers, and a plausibility norming task was employed consisting of 39 pairs of test items. In the first task, the subjects were asked to choose from a scale of five levels ranked from "extremely unnatural" to "extremely natural." Four factors were taken into account - overtness, salience of syntactic position, uniqueness and word- 
types. The quantifiers used in the experiment were every, no and many. The purpose of the norming task was to test the uniqueness of the antecedent.

The results showed four different effects. A prenominal-postnominal effect where acceptability in the postnominal type was higher indicated that the salience of syntactic position did work. An effect of overtness was found, showing that the acceptability of the overt types greatly exceeded the other types. There was also an effect of word-type in that the $\mathrm{N}$-owner type was much more acceptable than the N-less type, and an effect of uniqueness was obtained where sentences with unique antecedents were rated higher than those with non-unique ones. Also, an interaction was found between uniqueness and the word-type where only the Nless type showed a uniqueness effect. All in all, it was argued by Grosz et al. that the presence of the Overt NP Constraint and the Salient Position Condition was verified. Moreover, the interaction between uniqueness and word-type confirmed the dynamic approach, which predicted that uniqueness would not operate in the $\mathrm{N}$-owner type. This was the evidence that brought Grosz et al. to propose that the dynamic approach suited donkey sentences well.

\subsection{Summary}

Generally, interpretations of the antecedent from world knowledge are crucial for the donkey pronoun (Geurts 2002, Foppolo 2009, Grosz et al. 2014). Nevertheless, to what extent world knowledge, as a factor, influences interpretations of donkey sentences was found to vary by these previous studies. Quantifiers are one crucial factor since existential quantifiers tend to lead to existential readings only, while universal quantifiers often entail universal readings but sometimes existential readings as well (Geurts 2002, Foppolo 2009). Moreover, properties of the NP antecedent, and the uniqueness of the donkey pronoun and the antecedent are also important (Geurts 2002, Grosz et al. 2014).

However, these studies mainly focused on the generalizations of donkey pronouns (Geurts 2002, Foppolo 2009) and the referential properties of the antecedents (Grosz et al. 2014). Concerning the subject pool, all of them recruited only one group without a contrast group. With regard to the tasks, Geurts (2002) employed only one task, while Foppolo (2009) and Grosz et al. (2014) conducted two; moreover, the effect of context on test sentences was only examined by Foppolo (2009).

All in all, with interpretations of donkey sentences as a main concern, there has emerged no consensus regarding the generalizations of quantifier entailing interpretations. In addition, context, which influences interpretations, deserves to be further examined. In what follows, we will introduce the subjects and the research design of the present study. 


\section{Research design}

\subsection{Subjects}

The present study compares donkey constructions with bare conditionals from an empirical perspective. Few studies have investigated these two constructions in Mandarin Chinese experimentally or the role of quantification, which has been shown to be crucial in their interpretation. Thus, the present study aims to look into how quantifiers are interpreted in children's acquisition.

Since the work of Inhelder \& Piaget (1958) on children's interpretations of quantifiers, it has been found that children have difficulty obtaining adult-like interpretations of quantificational sentences like "Is every boy riding an elephant?" in response to a picture illustrating four elephants, three of which are being ridden by a boy. Adults tended to answer 'yes' while children between four and seven answered 'no, since they had difficulty comprehending such a mismatch of quantification in pictures (Herburger 1997, Cohen 2001, Geurts 2003). Following previous studies, we were interested to see whether this age difference comprehension difficulty would be evident when the experiment was concerned with donkey sentences and bare conditionals in Mandarin Chinese that are involved in quantification.

Accordingly, the present study, taking subjects within the age span of four to seven, set out to explore whether kindergarten children (KS) at five years of age would misinterpret the sentences and whether an adult-like interpretation would be obtained by seven years of age, Grade 2, or if this would not be acquired until Grade 4, that is, nine years of age. Therefore, three experimental groups comprising 18 five year olds, 18 seven year olds and 18 nine year olds were recruited to compare their interpretations of quantification in donkey sentences and bare conditionals. The KS subjects were Taiwanese children studying in a public kindergarten, while the Grade 2 and Grade 4 subjects were from an elementary school. A total of 18 native Mandarin-speaking adults aged 19 to 22 were recruited as a control group to contrast the overall performances of the child subjects.

\subsection{Materials and method}

This present study followed the essence of previous designs (Geurts 2002, Foppolo 2009) in which cross-sectional experiments were conducted and picture illustrations were provided as a Truth-Value Judgment task, and in another phase of the experiment, biasing contexts were provided prior to the target sentences. Unlike previous research (Geurts 2002, Foppolo 2009), in order to avoid picture ambiguity, the present study designed two picture situations for the subjects to 
choose from instead of four pictures. Previous studies have not examined the interrelationship in semantic interpretations between donkey sentences and bare conditionals, and no consensus on whether Chinese conditionals can be regarded as one type of donkey sentence has been reached (Cheng \& Huang 1996, Pan \& Jiang 1997, Cheung 2007, Wang 2007). Two tasks were conducted - one with sentences in isolation to see whether the subjects gave unanimous interpretations, and the other with sentences in context. Unlike Foppolo (2009), who included only biasing contexts in the task, we designed both supporting and biasing contexts to see whether the interpretations would be affected by context type.

\subsubsection{Donkey sentences}

Two tasks were designed: one with donkey sentences in isolation and the other with them in context. Illustrations of pictures and contexts were shown with biasing or supporting information. Interpretations of the sentences (universal or existential readings) were examined in order to explore the readings of donkey sentences and to compare them with readings of bare conditionals. The quantifiers in the design were mei 'every', youxie 'some' and bushi meige 'not every'. According to Krifka (1996), both every and not every yield a universal reading. Nevertheless, the reading of bushi meige 'not every' was an existential reading like youxie 'some' in Chinese, while mei 'every' was the quantifier eliciting a universal reading. Table 1 presents an example of a donkey sentence in insolation, abbreviated as DSII.

Table 1. An example of a donkey sentence in isolation

\begin{tabular}{l|l|l}
\hline The subject saw: & \multicolumn{2}{l}{ The subject heard: } \\
\hline \hline & "Meige you qiqiu de xiaopengyou \\
dou xihuan qiqiu." Qingwen shi \\
Tupian A haishi Tupian B shi zhe \\
ju hua de yisi ne? \\
"Every child who has a balloon \\
loves it." Is it Picture A or Picture B \\
that describes the meaning of this \\
sentence?"
\end{tabular}

An example of a donkey sentence in context, abbreviated as DSIC, is illustrated in Table 2. 
Table 2. An example of a donkey sentence in context

The subject saw:

\section{Scene 1:}

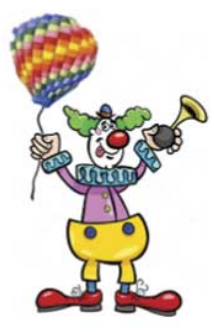

Scene 2:

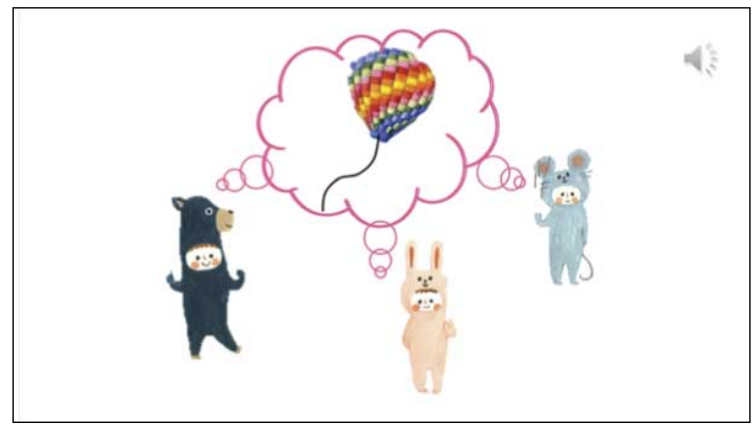

Scene 3:

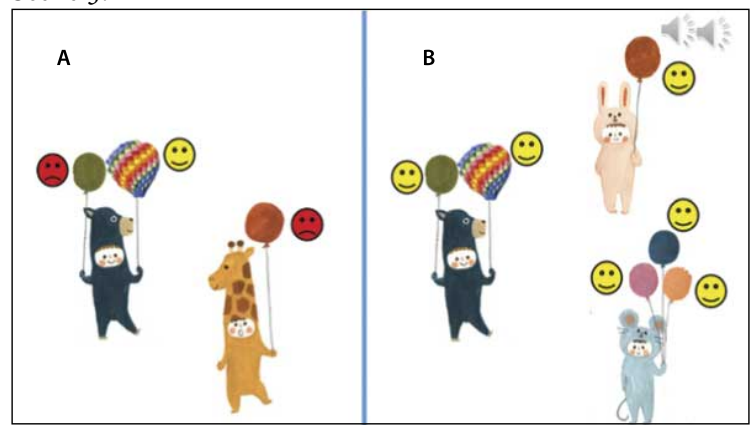

The subject heard:

Nongchang de xiaochou hen hui zuo qiqiu. Jintian ta zuo le yige tebie zaoxing de qiqiu.

'The clown in the amusement park is very good at making balloons. Today he made a special balloon.'

Xiaopengyou dou hen xiangyao na yige qiqiu.

'The children all want that balloon very much.'

"Meige you qiqiu de xiaopengyou dou xihuan qiqiu." Qingwen shi Tupian A haishi Tupian B shi zhe ju hua de yisi ne?

" "Every child who has a balloon loves it." Is it Picture A or Picture B that describes the meaning of this sentence?'

\subsubsection{Bare conditionals}

Two tasks were designed as well, one with bare conditionals in isolation and the other with them in context. Picture illustrations and contexts were provided with biasing or supporting interpretation. Below is an example of a bare conditional in isolation, abbreviated as BCII: 
Table 3. An example of a bare conditional in isolation

\begin{tabular}{|l|l|l}
\hline The subject saw: & The subject heard: \\
\hline \hline A & B Ni xihuan shei, wo jiu jian shei." \\
Qingwen shi Tupian A haishi \\
Tupian B shi zheju hua de yisi ne? \\
"I will meet whom you like." Is it \\
Picture A or Picture B that \\
describes the meaning of this \\
sentence?"
\end{tabular}

An example of a bare conditional in context, abbreviated as BCIC, is illustrated in Table 4.

Table 4. An example of a bare conditional in context

The subject saw:

The subject heard:

Xiaohua xihuan tade hao pengyou.

'Xiaohua likes her good friend.'

Scene 2:

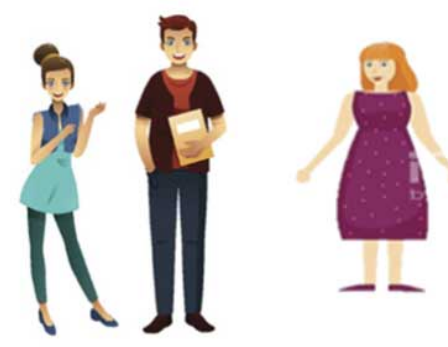

Xiaohua qing hao pengyou dao jiali wan rang mama renshi. 'Xiaohua invites her good friend home to let her mom get to know him.' 
The subject heard:

Scene 3:

Yinwei Mama shuo, "Ni xihuan
shei, wo jiu jian shei." Qingwen shi
Tupian A haishi Tupian B shi zheju
hua de yisi ne?
abecause Xiaohua's mother said
to her, "I meet whomever you
like." Is it Picture A or Picture B
that describes the meaning of this
sentence?"

\subsection{Procedure}

Before the experiments began, the task items of sentences in isolation and sentences in context were randomized. Tasks of sentences in isolation (DSII and BCII) were compiled into one file, and those of sentences in contexts (DSIC and BCIC) were treated likewise. All the task items were presented in the Truth-Value Judgment format with two pictures from which the subjects had to choose one, all of which were illustrated on PPT slides with the audio recording of the task items. Moreover, consent forms were collected to ensure the parents of the child subjects and adults had agreed to participate in the experiments.

As a preliminary step, the researchers held a training session at the beginning to familiarize the subjects with the task format. For each subject, there were two visits for data collection. In the first visit, the subjects took about 30 minutes to complete the DSII and BCII, and in the second visit, they spent about sixty minutes on DSIC and BCIC.

After the experiments, the mean scores of universal and existential readings indicated by each subject were counted. If a given test sentence was assigned to elicit a universal reading, but the selected picture demonstrated a universal reading, then one point was given. On the other hand, if the picture describing an existential reading was chosen, then no point was given. The data were analyzed with RStudio for comparisons of mean scores. The common analysis for data with comparisons between mean scores is the one-way ANOVA test, and the basic assumptions of the test are that the residuals of the data should be in normality and the data should have constant variance. Nevertheless, the amount of data generated by the present study was relatively small, and after transformation, the residuals of the data still violated normality and the data did not have constant variance. As a result, the one-way ANOVA test was not suitable for analysis. 
Instead, the Kruskal-Wallis rank sum test, which does not share the assumptions of the ANOVA test, was conducted to calculate the mean scores within each group and each type to see if there was a significant difference.

\section{Results and discussion}

\subsection{Relatedness of the two constructions}

In both constructions, readings were elicited and categorized. The donkey sentences were coded on the basis of the quantifier types in accordance to Kanazawa's Generalization (1994), where sentences with the quantifier mei 'every' were encoded with universal readings and sentences with the quantifier youxie 'some' and bushi meige 'not every' with existential readings. Bare conditionals were coded based on Cheng \& Huang (1996) with universal readings. The mean scores of the two constructions were counted in order to investigate their relatedness. Therefore, it is hypothesized that if the mean scores of the two constructions were close, the relatedness of the two constructions could be supported.

An overall between-construction comparison is presented in Figure 1.

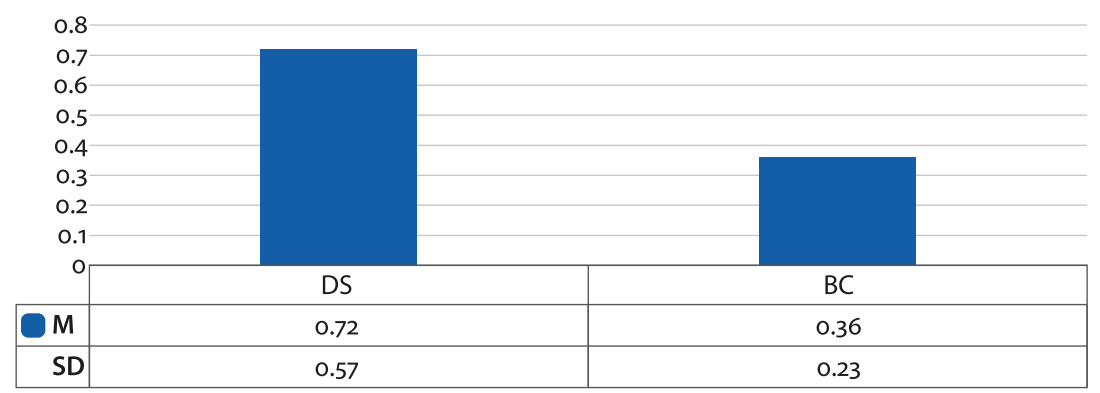

Figure 1. Subjects' overall interpretations of the two constructions

It was found that the subjects scored higher on the donkey sentences than on the bare conditionals $(M=0.72>0.36)$, indicating that the donkey sentences were much easier to interpret than the bare conditionals.

The Kruskal-Wallis rank sum test was used to examine the difference between the mean scores in the two constructions within the four groups, and a significant difference was found $\left(\chi^{2}(1)=77.83, p<.001\right)$. Table 5 examines more thoroughly the relatedness of the two constructions in each group. It was found that except for KS, which produced a higher score on bare conditionals $(M=0.66>0.43)$, Grade 2, Grade 4 and the adults all scored higher on the donkey sentences (Grade 2: $M=0.76>0.51$; Grade 4: $0.80>0.54$; Adults: $0.90>0.55$ ). The Kruskal- 
Wallis rank sum test showed that the differences between the two constructions in each group were significant (KS: $\chi^{2}(1)=13.06, p<.001$; Grade 2: $\chi^{2}(1)=37.77$, $p<.001$; Grade $4: \chi^{2}(1)=38.51, p<.001$; Adults: $\left.\chi^{2}(1)=92.27, p<.001\right)$.

Table 5. Each group's interpretations of the two constructions

\begin{tabular}{lccccccc}
\hline & Construction & \multicolumn{2}{c}{ DS } & & \multicolumn{2}{c}{ BC } & \\
\cline { 2 - 3 } Group & M & SD & & M & SD & $p$-value \\
\hline KS & 0.43 & 0.41 & & 0.66 & 0.22 & 0.0003019 \\
Grade 2 & 0.76 & 0.33 & & 0.51 & 0.20 & $7.949 \mathrm{E}-10$ \\
Grade 4 & 0.80 & 0.29 & & 0.54 & 0.27 & $5.444 \mathrm{E}-10$ \\
Adults & 0.90 & 0.16 & & 0.55 & 0.19 & $<2.2 \mathrm{E}-16$ \\
\hline
\end{tabular}

Moreover, age differences were found for each construction. With respect to the donkey sentences, from the mean scores of each group, a developmental pattern could be seen where the adults obtained the highest mean score $(M=0.90)$, followed by Grade $4(M=0.80)$, Grade $2(M=0.76)$ and $\mathrm{KS}(M=0.43)$. This overall between-group difference was significant $\left(\chi^{2}(3)=80.25, p<.001\right)$. Nevertheless, regarding the bare conditionals, KS $(M=0.66)$ had the highest mean score, while the older children (Grade 2 and Grade 4 ) and the adults produced roughly the same mean scores (Grade 2: $M=0.51$; Grade 4: $M=0.54$; Adults: $M=0.55$ ). This between-group difference was found to be significant as well $\left(\chi^{2}(3)=17.48\right.$, $p<.001)$.

Due to the fact that overall between-group differences for the two constructions were found to be significant, two-sample Wilcoxon tests were conducted for pairwise comparisons. The results are shown in Table 6.

Table 6. The between-group differences in DS and BC

\begin{tabular}{lcccccccc}
\hline Construction & \multicolumn{2}{c}{$\mathrm{DS}\left(\chi^{2}(3)=80.25, p<.001\right)$} & & \multicolumn{2}{c}{$\mathrm{BC}\left(\chi^{2}(3)=17.48, p<.001\right)$} \\
\cline { 2 - 4 } \cline { 7 - 9 } & KS & Grade 2 & Grade 4 & & KS & Grade 2 & Grade 4 \\
\hline Grade 2 & $9.678 \mathrm{E}-09$ & - & - & & $5.781 \mathrm{E}-05$ & - & - \\
Grade 4 & $1.325 \mathrm{E}-10$ & 0.395 & - & & 0.004697 & 0.5971 & - \\
Adults & $3.092 \mathrm{E}-16$ & 0.004136 & 0.04207 & & 0.003365 & 0.192 & 0.5758 \\
\hline
\end{tabular}

Note: The adjustment of $p$-values for the six paired comparisons was .01. ${ }^{5}$

5. Since there were six pairwise comparisons - KS vs. Grade 2, KS vs. Grade 4, KS vs. Adults, Grade 2 vs. Grade 4, Grade 2 vs. Adults and Grade 4 vs. Adults - the adjusted $p$-value was .05/ $6=.0084$, and rounding it to the nearest hundredth was .o1. Hence, if the result was a $p$-value of .04 , it was counted as insignificant. 
Concerning the donkey sentences, the differences between Grade 2 and Grade $4(p=.40)$ and between Grade 4 and the adults $(p=.04)$ were found to be insignificant, while the differences between KS and Grade 2, between KS and Grade 4, between KS and the adults, and between Grade 2 and the adults were all significant $(p<.01)$. In terms of the bare conditionals, the two-sample Wilcoxon tests identified significant differences among the comparisons between KS and Grade 2, between KS and Grade 4, and between KS and the adults $(p<.01)$. However, Grade 2, Grade 4 and the adults did not show any significant difference for comparisons made between one another (Grade 2-Grade 4: $p=.60$; Grade 2-Adults: $p=.19$; Grade 4-Adults: $p=.58$ ).

Accordingly, the KS group produced lower mean scores and showed great differences between them and the older groups regarding donkey sentences. Though the mean score of Grade 4 was higher than that of Grade 2, no significant difference was found between the two groups. Moreover, there was no significant difference between Grade 4 and the adults, indicating that they had acquired an adult-like interpretation. Regarding bare conditionals, Grade 2, Grade 4 and the adults tended to interpret them with more existential readings, but the KS group, in contrast, was not in the same vein as the older groups. This illustrated that the older groups had a unanimous tendency of not assigning an absolute reading to bare conditionals whereas those in the KS group had not yet acquired this tendency.

All the subjects' mean scores on the two constructions differed; hence, it can be inferred that the difficulty levels of the two constructions are not the same in that the subjects found the donkey sentences less challenging to interpret than the bare conditionals. Accordingly, it can be concluded that donkey sentences and bare conditionals are not derived from the same construction in terms of readings. Further investigation of the between-construction comparison for each group revealed that all four groups also considered the two constructions to have differing degrees of universal and existential readings. This finding can be explained by a syntactic approach.

It has been argued in the literature that the two constructions are related due to the fact that they both involve referentiality (Cheng \& Huang 1996, Pan \& Jiang 1997). For a donkey sentence, the pronoun can refer back to its antecedent, and for a bare conditional, shei 'who' in its consequent clause can also be anaphoric to the first shei 'who' in its antecedent clause. Hence, it is believed that the approach applied to donkey sentences can also account for bare conditionals. Cheng \& Huang (1996) argue that the unselective binding approach can be used to analyze both donkey sentences and bare conditionals, and such an approach leads bare conditionals to have universal readings. However, roughly only 0.50 of the mean scores on bare conditionals for Grade 2, Grade 4 and the adults indicate that half 
the sentences were interpreted with universal readings and half with existential readings. Hence, no clear tendency was found for either universal or existential readings, which is contrary to Cheng \& Huang (1996). Grade 2, Grade 4 and the adults, on the other hand, showed a clear tendency by interpreting donkey sentences based on quantifier types, in support of the literature (Kanazawa 1994, Krifka 1996, Geurts 2002, Foppolo 2009). Hence, in terms of readings, the two constructions are not identical, indicating that the approach adopted for donkey sentences cannot be utilized to examine bare conditionals in Chinese. Hence, we argue against Cheng \& Huang's (1996) analysis that the two constructions are related. Instead, our findings support Wang (2007), who considers that bare conditionals are one type of relative clause, whereas donkey sentences are not.

The overall within-group comparison between the constructions illustrates that the subjects scored significantly higher on the donkey sentences than on the bare conditionals, indicating that the donkey sentences were less challenging. Due to the fact that donkey sentences exhibit only one reading, either universal or existential, they are regarded as being far from obscure (Kanazawa 1994, Champollion 2016). Bare conditionals, in contrast, contain wh-indefinites that need context to be explicitly interpreted (Dekker 2001, Herburger 2015); therefore, they may exhibit more than one reading. Ambiguity makes this type of sentence more difficult to interpret.

In addition, a developmental pattern was found for donkey sentences where children above second grade (i.e., older than seven years of age) had an adult-like intuition. This supports the literature (Inhelder \& Piaget 1958, Herburger 1997, Cohen 2001, Geurts 2003), which states that children between the ages of four and seven have difficulties interpreting quantificational sentences. Also, according to the Stages of Cognitive Development proposed by Piaget (1936), children between seven and eleven are at a concrete operational stage where they start thinking logically. Hence, it is not until seven years of age that children no longer find it a challenge to interpret quantification.

Nevertheless, concerning bare conditionals, except for the KS group, overall the older groups showed no differences in interpreting bare conditionals. The indefiniteness of a $w h$-word in a bare conditional is derived from its question form (Haspelmath 1997, Thuan \& Bruening 2013). It has been found that additional derivations in word forms occur with an indefiniteness use derived from the question use; therefore, the question use is said to be the default form while the indefinite use is a derived one. In the process of acquisition, children must learn the default use first (i.e., the question use of $w h$-words) and the indefinite use later. Accordingly, it can be inferred that those in the KS group were still in the process of applying the default use of $w h$-words, leading to the incongruity between them and the older groups. 


\subsection{Contextual effect}

An overall comparison between context types is presented in Figure 2, where the subjects scored highest on sentences in a supporting context, scored second on sentences in isolation, and scored the lowest on sentences in a biasing context $(M=0.76>0.66>0.59)$.

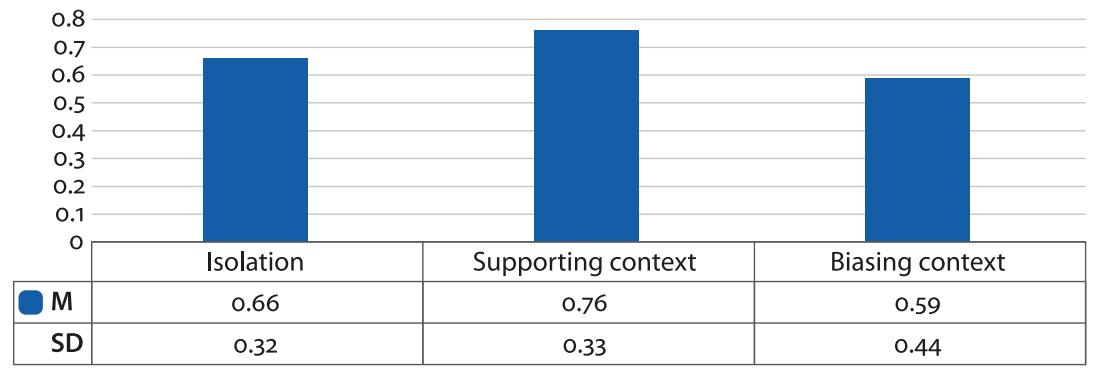

Figure 2. Subjects' overall interpretations of the context types

Using the Kruskal-Wallis rank sum test, an overall between-type difference within the four groups was found $\left(\chi^{2}(2)=32.90, p<.001\right)$. The two-sample Wilcoxon tests were used to examine pairwise comparisons; hence, as can be seen in Table 7, the comparisons between sentences in isolation and in supporting contexts, and between sentences in supporting and biasing contexts were found to be significant $(p<.02)$.

Table 7. The overall between-type differences

\begin{tabular}{lccc}
\hline Context & $\begin{array}{c}\text { Isolation - supporting } \\
\text { context }\end{array}$ & $\begin{array}{c}\text { Isolation - biasing } \\
\text { context }\end{array}$ & $\begin{array}{c}\text { Supporting context - biasing } \\
\text { context }\end{array}$ \\
\hline$p$-value & $1.808 \mathrm{E}-07$ & 0.3489 & $1.591 \mathrm{E}-06$ \\
\hline
\end{tabular}

Note: The adjustment of $p$-values for the three paired comparisons was .02. ${ }^{6}$

The effect of different context types on the donkey sentences and bare conditionals was investigated. Table 8 provides the between-type comparisons for the donkey sentences.

As seen in Table 8, the mean scores of KS, Grade 2 and Grade 4 subjects were higher in both supporting and biasing contexts than the scores on sentences

6. Three pairwise comparisons - in isolation vs. in a supporting context, in isolation vs. in a biasing context, and in a supporting context vs. in a biasing context - were needed; therefore, the adjusted $p$-value was $.05 / 3=.0167$, and rounding it to the nearest hundredth was .02. Hence, if the result were a $p$-value of .o4, it was counted as insignificant. 
Table 8. Each group's interpretations of DS in the three context types

\begin{tabular}{|c|c|c|c|c|c|c|c|}
\hline \multirow{2}{*}{ Group } & \multicolumn{2}{|c|}{ Isolation } & \multicolumn{2}{|c|}{ Supporting context } & \multicolumn{2}{|c|}{ Biasing context } & \multirow[b]{2}{*}{$p$-value } \\
\hline & $\mathbf{M}$ & SD & M & SD & $\mathbf{M}$ & SD & \\
\hline KS & 0.39 & 0.41 & 0.48 & 0.46 & 0.48 & 0.49 & 0.5722 \\
\hline Grade 2 & 0.75 & 0.34 & 0.81 & 0.33 & 0.81 & 0.38 & 0.1554 \\
\hline Grade 4 & 0.80 & 0.30 & 0.92 & 0.23 & 0.85 & 0.33 & 0.005361 \\
\hline Adults & 0.95 & 0.14 & 0.97 & 0.12 & 0.89 & 0.27 & 0.1639 \\
\hline
\end{tabular}

in isolation. The KS and Grade 2 subjects did not show significant differences among the three contextual types (KS: $\chi^{2}(2)=1.12, p=.57$; Grade $2: \chi^{2}(2)=3.72$, $p=.16$ ), while Grade 4 was the only group that did show a significant difference $\left(\chi^{2}(2)=10.46, p<.01\right)$. Since the mean score of Grade 4 subjects greatly increased in supporting contexts, pairwise between-type comparisons for Grade 4 indicated that a significance was found in the isolation-supporting context comparison $(p<.02)$. The adults, on the other hand, gained a higher mean score on sentences with a supporting context only, but a lower score on those with a biasing context; however, the between-type differences were not significant $\left(\chi^{2}(2)=3.62, p=.16\right)$. Between-group comparisons for each contextual type are shown in Table 9.

Table 9. The between-group differences for DS in the three context types

\begin{tabular}{|c|c|c|c|c|c|c|c|c|c|}
\hline \multirow[t]{2}{*}{ Context } & \multicolumn{3}{|c|}{$\begin{array}{c}\text { Isolation } \\
\left(\chi^{2}(3)=57.71, p<.001\right)\end{array}$} & \multicolumn{3}{|c|}{$\begin{array}{c}\text { Supporting context } \\
\left(\chi^{2}(3)=55.76, p<.001\right)\end{array}$} & \multicolumn{3}{|c|}{$\begin{array}{c}\text { Biasing context } \\
\left(\chi^{2}(3)=31.05, p<.001\right)\end{array}$} \\
\hline & $\mathrm{KS}$ & Grade 2 & Grade 4 & KS & Grade 2 & Grade 4 & KS & Grade 2 & Grade 4 \\
\hline Grade 2 & $8.347 \mathrm{E}-06$ & - & - & $8.092 \mathrm{E}-05$ & - & - & 0.0002902 & - & - \\
\hline Grade 4 & $8.465 \mathrm{E}-07$ & 0.5954 & - & $8.017 \mathrm{E}-08$ & 0.05622 & - & $2.856 \mathrm{E}-05$ & 0.5841 & - \\
\hline Adults & $3.466 \mathrm{E}-12$ & 0.0004808 & 0.001884 & $4.591 \mathrm{E}-10$ & 0.001689 & 0.1766 & $3.721 \mathrm{E}-06$ & 0.3587 & 0.7211 \\
\hline
\end{tabular}

Note: The adjustment of $p$-values for the six paired comparisons was .o1.

Significant differences were found in the between-group comparisons of sentences in isolation $\left(\chi^{2}(3)=57.71, p<.001\right)$, in supporting contexts $\left(\chi^{2}(3)=55.76\right.$, $p<.001)$, and in biasing contexts $\left(\chi^{2}(3)=31.05, p<.001\right)$. The two-sample Wilcoxon tests reported that the three groups of children interpreted sentences in isolation significantly differently from the adults $(p<.01)$. The KS group showed significant differences from the two older groups respectively $(p<.01)$, while the Grade 2-Grade 4 comparison did not show significance $(p=.60)$. In supporting contexts, Grade 2 and Grade 4 did not show a significant difference $(p=.06)$, and only Grade 4 showed no significant difference from the adults $(p=.18)$, while Grade 2 and KS did $(p<.01)$. In biasing contexts, significant differences were 
found between the KS and older groups $(p<.01)$, but no difference was found between Grade 2 and Grade $4(p=.58)$, between Grade 2 and the adults $(p=.36)$ and between Grade 4 and the adults $(p=.72)$.

Now, let's turn to the effects on bare conditionals as shown in Table 10, which shows the between-type comparisons.

Table 10. Each group's interpretations of BC in the three context types

\begin{tabular}{|c|c|c|c|c|c|c|c|}
\hline \multirow[b]{2}{*}{ Group } & \multicolumn{2}{|c|}{ Isolation } & \multicolumn{2}{|c|}{$\begin{array}{c}\text { Supporting } \\
\text { context }\end{array}$} & \multicolumn{2}{|c|}{$\begin{array}{l}\text { Biasing } \\
\text { context }\end{array}$} & \multirow[b]{2}{*}{$p$-value } \\
\hline & M & SD & M & SD & M & SD & \\
\hline KS & 0.64 & 0.22 & 0.66 & 0.24 & 0.71 & 0.32 & 0.237 \\
\hline Grade 2 & 0.54 & 0.20 & 0.65 & 0.27 & 0.26 & 0.29 & $2.102 \mathrm{E}-07$ \\
\hline Grade 4 & 0.56 & 0.25 & 0.69 & 0.32 & 0.29 & 0.28 & $1.756 \mathrm{E}-06$ \\
\hline Adults & 0.65 & 0.21 & 0.85 & 0.15 & 0.09 & 0.14 & $<2.2 \mathrm{E}-16$ \\
\hline
\end{tabular}

Except for the KS group, who received the highest mean score on sentences in biasing contexts, the older groups of children and the adults all produced the highest mean scores on bare conditionals in supporting contexts, followed by sentences in isolation and those in biasing contexts. The differences of mean scores were found insignificant for the KS group $\left(\chi^{2}(2)=2.88, p=.24\right)$, suggesting that preschoolers are still at a sentence level where decontextualized speech is often uttered especially when syntactically complex utterances are expressed (Tannen 1982). However, the statistical differences were significant for the Grade 2 group, the Grade 4 group and the adults (Grade 2: $\chi^{2}(2)=30.75, p<.001$; Grade 4: $\chi^{2}(2)=26.50, p<.001$; Adults: $\chi^{2}(2)=78.45, p<.001$ ). Accordingly, two-sample Wilcoxon tests were used to explore their differences, as can be seen in Table 11.

Table 11. The between-type differences for BC in each group

\begin{tabular}{|c|c|c|c|}
\hline Group & $\begin{array}{c}\text { Isolation - } \\
\text { supporting context }\end{array}$ & $\begin{array}{l}\text { Isolation - biasing } \\
\text { context }\end{array}$ & $\begin{array}{l}\text { Supporting context - } \\
\text { biasing context }\end{array}$ \\
\hline Grade 2 & 0.04965 & $1.083 \mathrm{E}-05$ & $1.089 \mathrm{E}-06$ \\
\hline Grade 4 & 0.06565 & $8.287 \mathrm{E}-05$ & $3.852 \mathrm{E}-06$ \\
\hline Adults & $7.147 \mathrm{E}-05$ & $4.954 \mathrm{E}-13$ & $4.8 \mathrm{E}-14$ \\
\hline
\end{tabular}

Note: The adjustment of $p$-values for the three paired comparisons was .02.

All three groups showed significant differences in the comparison between isolation and biasing contexts and between supporting and biasing contexts $(p<.02)$. The adults also showed a significant between-type difference in the com- 
parison between scores on sentences in isolation and on sentences with a supporting context $(p<.02)$.

The pairwise between-group comparisons in sentences of the three contextual types is shown in Table 12.

Table 12. The between-group differences for BC in the three context types

\begin{tabular}{|c|c|c|c|c|c|c|c|c|c|}
\hline \multirow{2}{*}{ Context } & \multicolumn{3}{|c|}{$\begin{array}{c}\text { Isolation } \\
\left(\chi^{2}(3)=8.01, p=.05\right)\end{array}$} & \multicolumn{3}{|c|}{$\begin{array}{l}\text { Supporting context } \\
\left(\chi^{2}(3)=14.49, p<.01\right)\end{array}$} & \multicolumn{3}{|c|}{$\begin{array}{c}\text { Biasing context } \\
\left(\chi^{2}(3)=56.16, p<.001\right)\end{array}$} \\
\hline & KS & $\mathrm{rad}$ & rade 4 & KS & Grade 2 & Grade 4 & KS & Grade 2 & Grade 4 \\
\hline Grade 2 & - & - & - & 0.8456 & - & - & $3.249 \mathrm{E}-07$ & - & - \\
\hline Grade 4 & $4-$ & - & - & 0.5023 & 0.4518 & - & $1.932 \mathrm{E}-06$ & 0.4962 & - \\
\hline Adults & - & - & - & 0.0002297 & 0.000494 & 0.05799 & $2.572 \mathrm{E}-11$ & 0.00916 & 0.0006525 \\
\hline
\end{tabular}

Note: The adjustment of $p$-values for the six paired comparisons was .o1.

First, it was found that comparisons of mean scores in isolation were insignificant $\left(\chi^{2}(3)=8.01, p=.05\right)$. However, a significant discrepancy was found in the scores for sentences in supporting contexts $\left(\chi^{2}(3)=14.49, p<.01\right)$. Two-sample Wilcoxon tests showed that, compared with the adults, the KS and Grade 2 groups each showed significant differences $(p<.01)$, but the Grade 4 group did not $(p=.06)$, and that the three experimental groups did not show a significant difference between one another (KS-Grade 2: $p=.85 ;$ KS-Grade 4: $p=.50$; Grade 2-Grade 4: $p=.45$ ). For sentences in biasing contexts, a significance difference was found in the overall between-group comparison $\left(\chi^{2}(3)=56.16, p<.001\right)$. Pairwise between-group comparisons indicated that the Grade 2 and Grade 4 groups did not display a significant difference $(p=.50)$, but comparisons between the other groups did $(p<.01)$.

Previous studies have proposed that the context can influence the interpretation of sentences with respect to the syntax-semantic interface (Prévost \& Paradis 2004, Foppolo 2009, Ireri et al. 2012). Lin \& Chan (2019) also found that context flexibly influenced conceptual processing. In the present study, contextual effects on both donkey sentences and bare conditionals were found. Although concepts of quantification are abstract, providing context can offer children both clues and time to process sentences. Nevertheless, the two constructions exhibit different degrees of explicitness in quantification, that is, wh-words in bare conditionals are less explicit than quantifier types in donkey sentences. Hence, different degrees of explicitness render different degrees of contextual effect, resulting in there being more significant effects on donkey sentences than on bare conditionals.

In donkey sentences, it was found that sentences in context helped the older groups of children gain higher mean scores than did sentences in isolation. In 
both the supporting and the biasing context, the older children interpreted donkey sentences more accurately. It can be reasoned that the memory spans of children are limited; however, with contexts lengthening the span of time in which to figure out the situations of the tasks, children can then make better interpretations with their processing devices working more properly to interpret quantificational sentences. In this case, context suffices for interpreting vague concepts (DeVault \& Stone 2004); hence, children can answer donkey sentences better.

In contrast, $w h$-words in bare conditionals are not as explicit as quantifiers in donkey sentences. Bare conditionals themselves are implicit and ambiguous so they cannot simply be resolved by the provision of context. As found in the mean scores of the Grade 2, Grade 4 and the adult groups, their interpretations of bare conditionals were quite flexible and easily influenced by context. It is worth noting that the adults' mean scores in both supporting and biasing contexts were highly affected by context. Since the expected readings for all bare conditionals were universal readings, the impact of context rendered a high mean score on sentences in a supporting context but a very low score on those in a biasing context. This difference can also be found in the older group of children though its mean scores did not display as huge a difference as did the adult group. It can be reasoned that Chinese is more a discourse-oriented language (Huang 1984, Ni 1987) and that readings of bare conditionals are more a pragmatic matter (Dekker 2001, Herburger 2015) where readings fluctuate on the basis of context; hence, when a factor determining the reading of a sentence is implicit and unpredictable, context can easily influence its reading.

In sum, context was certainly a crucial factor that helped the subjects obtain readings more easily, both in positive and negative contexts, yet the explicitness of the operator that assigns a reading to a sentence matters. If the operator is explicit enough, like quantifiers, context functions as a synergy, enhancing mean scores and making sentences more easily interpreted. However, if the operator, like $w$ h-words, is implicit, context will then dominate readings and children will thus be easily influenced by contextual clues.

As for the developmental pattern for the contextual effects, the results show that the children over seven years old were keen to pick up on contextual clues; hence, they interpreted sentences with the influence of context. The children under seven years old were not aware of contextual clues (Yatsushiro 2008) since they were still at the stages of learning and producing discourse (Pearson \& de Villiers 2005). However, children above seven years of age can start appreciating context to infer readings of sentences. 


\subsection{Age effect}

Language acquisition is a process that develops gradually by age; hence, the issue of age has been widely discussed. The present study analyzes children's interpretations of sentences that are concerned with quantification and compares their behavior with that of adults in order to understand their pattern of acquisition. Figure 3 below presents three acquisition stages found by this study, and it also shows that our child subjects were indeed able to interpret donkey sentences and bare conditionals in Chinese, though their performance was not adult-like. This provides a piece of evidence for Chomsky's Innateness Hypothesis that that children have the innate ability to acquire a language without explicit teaching.

\section{Stage I (KS)}

1. Consider the two constructions as not related and have no adult-like intuition about DS or BC

2. Are insensitive to all three context types in DS

3. Show adult-like interpretations of $\mathrm{BC}$ in isolation

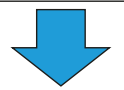

\section{Stage II (Grade 2)}

1. Consider the two constructions as not related, but with an adult-like intuition about BC but not about DS

2. Show adult-like interpretations of DS in biasing contexts

3. Show adult-like interpretations of BC in isolation

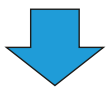

\section{Stage III (Grade 4)}

1. Consider the two constructions as not related, but have an adult-like intuition about DS and $\mathrm{BC}$

2. Show adult-like interpretations of DS in biasing and supporting contexts

3. Show adult-like interpretations of $\mathrm{BC}$ in isolation and in supporting contexts

Figure 3. Children's acquisition of donkey sentences and bare conditionals

At Stage I, the subjects were aware that donkey sentences and bare conditionals were not related, but they had not gained an adult-like pattern in interpreting these sentences. It has been found that children, especially those under seven years of age, have difficulties interpreting quantificational sentences (Herburger 1997, Cohen 2001, Geurts 2003). Even with context provided, the subjects was not sensitive to contextual clues and hence did not regard them as hints to interpret quantification. 
At Stage II, the subjects might have perceived that donkey sentences and bare conditionals were not related regarding readings, but they had only acquired an adult-like accuracy on bare conditionals. The subjects were at the stage of starting to be sensitive to context; hence, they had an adult-like intuition about donkey sentences in biasing contexts.

At Stage III, the subjects also showed an awareness that the two constructions were not so related, and they assigned an adult-like reading to both donkey sentences and bare conditionals. They were also conscious of contextual clues since their mean scores showed they were affected by supporting contexts of donkey sentences and bare conditionals and by biasing contexts of donkey sentences, their scores being similar to the mean scores of the adults. Overall, the subjects at this age had acquired most everything related to quantification, except the notion of youxie 'some, which remained not fully developed.

\section{Conclusion}

The present study explored children's first language acquisition of Chinese donkey sentences and bare conditionals in terms of their interpretation. Several factors were considered in the design of the study and the major findings are as follows.

First, in previous research (Cheng \& Huang 1996, Pan \& Jiang 1997, Wang 2007) the relatedness of the two constructions was discussed, but no consensus emerged. The present research, on the other hand, sheds some light on this issue from an empirical viewpoint. In this present study, donkey sentences were found to be less challenging to interpret than bare conditionals, and this discrepancy was found to be significant; hence, the two constructions might not have been perceived as related by our subjects. It can thus be inferred that quantifier types in donkey sentences do not display as much vagueness as do $w$ h-indefinites in bare conditionals. Second, contextual effects were found to be significant and affective to readings of both donkey sentences and bare conditionals. Context offered the subjects more time to ruminate about the readings; hence, whether it was supporting or biasing, the subjects scored higher on donkey sentences in context than on those sentences in isolation. On the other hand, context was an influential factor affecting the interpretations of bare conditionals as well but in a different way where the subjects' mean scores increased for sentences in a supporting context, but greatly decreased for those in a biasing context. Finally, a developmental pattern of children's interpretations was found where overall the KS group, children at around five years of age, had difficulty with quantification while Grade 2 subjects were in the middle of the process of acquiring quantification and Grade 4 
subjects had interpretations almost like those of the adults. Except for the reading of youxie 'some,' Grade 4 subjects had obtained an adult-like intuition about quantification of donkey sentences and bare conditionals.

Three limitations are recognized in the present study and it is recommended that they be taken into consideration for future research. First, the older groups of children and the adults did not show such a clear pattern in interpreting bare conditionals as they did in interpreting donkey sentences. Although this study followed previous research (Geurts 2002, Foppolo 2009) using acceptability tasks wherein the subjects had to choose from between two pictures, we can infer that the acceptability of the test sentences might be affected by grammaticality; hence, variations occurred in the readings. Moreover, the picture illustrations may have caused some ambiguity that biased the subjects' interpretations. As a result, future research could adopt grammaticality or interpretation tasks for children to complete to avoid these potential problems. Second, mean scores were counted based on the way it had been done in the previous literature, yet it would be more suitable to compare interpretations of sentence types on the basis of the test subjects' preferences for readings. Therefore, frequency counts together with the Chi-squared test could be used instead of mean scores for statistical analysis in future research. Finally, Grade 4 subjects were found unable to interpret youxie 'some' in an adult-like manner; hence, children over ten years of age should be recruited in future research to further investigate the developmental pattern of children's acquisition of sentences with quantification.

\section{Acknowledgements}

This work was financially supported by the "Chinese Language and Technology Center" of National Taiwan Normal University (NTNU) within the framework of the Higher Education Sprout Project by the Ministry of Education (MOE) in Taiwan.

\section{References}

Champollion, Lucas. 2016. Homogeneity in donkey sentences. Proceedings of the 26 th Semantics and Linguistic Theory Conference, SALT, ed. by Mary Moroney, Carol-Rose Little, Jacob Collard and Dan Burgdorf, 684-704. Austin, TX: University of Texas.

Cheng, Lisa Lai-Shen, and C.-T. James Huang. 1996. Two types of donkey sentences. Natural Language Semantics 4:121-163. https://doi.org/10.1007/BFoo355411 
Cheung, Candice Chi Hang. 2007. The syntax and semantics of bare conditionals in Chinese. Proceedings of Sinn und Bedeutung 11, ed. by Estela Puig-Waldmüller, 150-164. Barcelona: Universitat Pompeu Fabra.

Chomsky, Noam. 1986. Knowledge of Language: Its Nature, Origin and Use. New York: Praeger. Cohen, Ariel. 2001. Relative readings of many, often, and generics. Natural Language

Semantics 9.1:41-67. https://doi.org/10.1023/A:1017913406219

Cook, Vivian James. 1988. Chomsky's Universal Grammar: An Introduction. Oxford, UK: Blackwell.

Crain, Stephen, Rosalind Thornton, Carole Boster, Laura Conway, Diane Lillo-Martin, and Elaine Woodams. 2009. Quantification without quantification. Language Acquisition 5.2:83-153. https://doi.org/10.1207/s15327817la0502_2

Dekker, Paul. 2001. On if and only. Proceedings of the 11th Semantics and Linguistic Theory Conference, SALT, ed. by Rachel Hastings, Brendan Jackson and Zsofia Zvolenszky, 114-133. New York: New York University.

DeVault, David, and Matthew Stone. 2004. Interpreting vague utterances in context. Proceedings of the 2oth International Conference on Computational Linguistics, COLING, ed. by Lothar Lemnitzer, Detmar Meurers and Erhard Hinrichs, 1247-1253. Geneva, Switzerland: University of Geneva. https://doi.org/10.3115/1220355.1220536

Foppolo, Francesca. 2009. The puzzle of donkey anaphora resolution. Proceedings of the 38 th Annual Meeting of the North East Linguistic Society, ed. by Martin Walkow and Muhammad Abdurrahman, 297-310. Amherst, MA: University of Massachusetts.

Gelman, Susan A., and Henry M. Wellman. 1991. Insides and essences: Early understandings of the non-obvious. Cognition 38.3:213-244. https://doi.org/10.1016/0010-0277(91)90007-Q

Geurts, Bart. 2002. Donkey business. Linguistics and Philosophy 25.2:129-156. https://doi.org/10.1023/A:1014624331450

Geurts, Bart. 2003. Quantifying kids. Language Acquisition 11:197-218. https://doi.org/10.1207/s15327817la1104_1

Gopnik, Alison. 1988. Conceptual and semantic development as theory change: The case of object permanence. Mind \& Language 3.3:197-216.

https://doi.org/10.1111/j.1468-0017.1988.tboo143.x

Gopnik, Alison, and Andrew N. Meltzoff. 1997. Words, Thoughts, and Theories. Cambridge, MA: The MIT Press.

Grosz, Patrick G., Pritty Patel-Grosz, Evelina Fedorenko, and Edward Gibson. 2014. Constraints on donkey pronouns. Journal of Semantics 32.4:619-648. https://doi.org/10.1093/jos/ffuoo9

Haspelmath, Martin. 1997. Indefinite Pronouns. Oxford, UK: Clarendon Press.

Herburger, Elena. 1997. Focus and weak noun phrases. Natural Language Semantics 5.1:53-78. https://doi.org/10.1023/A:1008222204053

Herburger, Elena. 2015. Conditional perfection: The truth and the whole truth. Proceedings of the 25th Semantics and Linguistic Theory Conference, SALT, ed. by Sarah D'Antonio, Mary Moroney and Carol Rose Little, 615-635. Stanford, CA: Stanford University.

Huang, C.-T. James. 1984. On the distribution and reference of empty pronouns. Linguistic Inquiry 15.4:531-574.

Inhelder, Bärbel, and Jean Piaget. 1958. The Growth of Logical Thinking from Childhood to Adolescence: An Essay on the Construction of Formal Operational Structures. New York: Basic Books. https://doi.org/10.1037/10034-000 
Ireri, Anthony M., Daniel M. Mukuni, Philomena N. Mathuvi, Amos M. Njagi, and Njagi I. Karugu. 2012. An overview of major biological and contextual factors in language acquisition. American Journal of Linguistics 1.3:33-39.

Kanazawa, Makoto. 1994. Weak vs. strong readings of donkey sentences and monotonicity inferences in a dynamic setting. Linguistics and Philosophy 17.2:109-158. https://doi.org/10.1007/BFoo984775

Keil, Frank C. 1989. Concepts, Kinds, and Cognitive Development. Cambridge, MA: The MIT Press.

Krifka, Manfred. 1996. Pragmatic strengthening in plural predications and donkey sentences. Proceedings of the 6th Semantics and Linguistic Theory Conference, SALT, ed. by Teresa Galloway and Justin Spence, 136-153. Ithaca, NY: Cornell University.

Lin, Keng-yu, and Shiao-hui Chan. 2019. When senses meet functions: An amodal stage in conceptual processing. Journal of Cognitive Psychology 31.1:64-75. https://doi.org/10.1080/20445911.2018.1560299

$\mathrm{Ni}$, Weijia. 1987. Empty topics in Chinese. UConn Working Papers in Linguistics 1. Storrs, CT: University of Connecticut.

Pan, Haihua, and Yan Jiang. 1997. NP interpretation and donkey sentences in Chinese. Paper presented at the Workshop on Interface Strategies in Chinese, Cornell University, Ithaca.

Pearson, Barbara Zurer, and Peter de Villiers. 2005. Child language acquisition: Discourse, narrative, and pragmatics. Encyclopedia of Language and Linguistics (2nd edition), ed. by Keith Brown. Oxford: Elsevier.

Piaget, Jean. 1936. Origins of Intelligence in the Child. London: Routledge \& Kegan Paul. Prévost, Philippe, and Johanne Paradis (eds.) 2004. The Acquisition of French in Different Contexts: Focus on Functional Categories. Amsterdam: John Benjamins. https://doi.org/10.1075/lald.32

Tannen, Deborah. 1982. Spoken and Written Language: Exploring Orality and Literacy. Norwood, NJ: Ablex Publishing.

Thuan, Tran, and Benjamin Bruening. 2013. Wh-phrases as indefinites: A Vietnamese perspective. Linguistics of Vietnamese: An International Survey, ed. by Daniel Hole and Elisabeth Löbel, 217-241. Berlin: Mouton de Gruyter. https://doi.org/10.1515/9783110289411.217

Wang, Xin. 2007. Guanyu luziju de jidian yiwen yu sikao [Some questions and reflections about donkey sentences]. Yuwen Xuekan 2:69-72.

Yatsushiro, Kazuko. 2008. Quantifier acquisition: Presuppositions of "every". Proceedings of Sinn und Bedeutung 12, ed. by Atle Grønn, 663-677. Oslo: University of Oslo.

\title{
Address for correspondence
}

\author{
Chun-yin Doris Chen \\ Department of English \\ National Taiwan Normal University \\ Taipei, TAIWAN \\ chunyin@ntnu.edu.tw
}




\section{Co-author information}

Wei-ling Eileen Lin

Department of English

National Taiwan Normal University

Taipei, TAIWAN

eileen810810@gmail.com
Gerardo Fernandez-Salgueiro

Department of English

National Taiwan Normal University

Taipei, TAIWAN

gfsayang@ntnu.edu.tw

\section{Publication history}

Date received: 13 February 2019

Date revised: 13 June 2019

Date accepted: 18 December 2019 